Salah Drabla · Ziloukha Zellagui

\title{
Variational Analysis and the Convergence of the Finite Element Approximation of an Electro-Elastic Contact Problem with Adhesion
}

Received: 25 December 2010 / Accepted: 25 October 2011 / Published online: 2 December 2011

(C) The Author(s) 2011. This article is published with open access at Springerlink.com

\begin{abstract}
A model for the adhesive, quasi-static and frictionless contact between an electro-elastic body and a rigid foundation is studied in this paper. The contact is modelled with Signorini's conditions with adhesion. We provide variational formulation for the problem and prove the existence of a unique weak solution to the model. The proofs are based on arguments of time-dependent variational inequalities, differential equations and Banach fixed point. Then, a fully discrete scheme is introduced based on the finite element method to approximate the spatial variable and the backward Euler scheme to discretize the time derivatives. Error estimates are derived on the approximative solutions from which the linear convergence of the algorithm is deduced under suitable regularity conditions.
\end{abstract}

Keywords Piezoelectricity $\cdot$ Elasticity $\cdot$ Adhesion $\cdot$ Error estimates $\cdot$ Finite element method

Mathematics Subject Classification (2010) 35J85 · 49J40 - 47J20 · 74M15

\section{Introduction}

The piezoelectric effect is characterized by the coupling between the mechanical and electrical properties of the materials. Indeed, the apparition of electric charges on some crystals submitted to the action of body forces and surface traction was observed and their dependence on the deformation process was underlined. Conversely, it was proved experimentally that the action of electric field on the crystals may generate strain and stress. A deformable material which presents such a behavior is called a piezoelectric material. Piezoelectric materials

S. Drabla $(\bowtie)$

Department of Mathematics, Faculty of Sciences, University of Sétif, 19000 Setif, Algeria

E-mail: drabla_s@yahoo.fr

\section{Z. Zellagui}

University of Béjaia, 06000 Bejaia, Algeria

E-mail: z_zellagui@yahoo.fr 
are used extensively as switches and actuators in many engineering systems, in radioelectronics, electroacoustics, and measuring equipments. General models for electro-elastic materials can be found in $[4,7,13]$. In this work, we describe a model for the process of frictionless, adhesive, unilateral contact between an electro-elastic body and a rigid obstacle, the so-called foundation. Our purpose is to describe the delamination process when the friction tangential traction is negligible in comparison with the traction due to adhesion. We provide a variational formulation of the model and, using arguments of evolutionary equations in Banach spaces, prove that the model has a unique weak solution.

This work is a companion and an extension of the results in $[5,9,11]$, where the frictionless adhesion contact and contact between two frictionless elastic piezoelectric bodies are investigated when the contact is described by normal compliance. In addition, here we allow for the deterioration of the bonding process as cycles of bonding and debonding take place. Indeed, in such cases a deterioration in the bond strength might be observed and rebonded glue may not be as strong as a new one. Therefore, we allow for the dependence of the process on the bonding history.

The paper is structured as follows. In Sect. 2 we present a brief description of the mechanical model and provide its variational formulation in terms of the displacement field in Sect. 3. Then, we prove an existence and uniqueness result (Theorem 4.1) in Sect. 4. Finally, a fully discrete scheme is introduced in Sect. 5, based on the finite element method to approximate the spatial variable and the backward Euler scheme to discretize the time derivatives. A main error estimates result is proved, Theorem 6.1, from which the linear convergence of the algorithm is deduced under suitable regularity conditions.

\section{Problem Statement}

We consider the following physical setting. An electro-elastic body occupies a bounded domain $\Omega \subset \mathbb{R}^{d},(d=$ $2,3)$ with a smooth boundary $\Gamma=\partial \Omega$. The body is submitted to the action of body forces of density $f_{0}$ and volume electric charges of density $q_{0}$. It is also submitted to mechanical and electric constraints on the boundary. To describe them, we decompose $\Gamma$ either into three disjoint parts, $\Gamma_{1}, \Gamma_{2}$ and $\Gamma_{3}$, or into two disjoint parts, $\Gamma_{a}$ and $\Gamma_{b}$, such that meas $\left(\Gamma_{1}\right)>0$, meas $\left(\Gamma_{a}\right)>0$, and $\Gamma_{3} \subseteq \Gamma_{b}$. We assume that the body is in adhesive contact with an insulator foundation. We also assume that the electrical potential vanishes on $\Gamma_{a}$ and a surface electric charge of density $q_{2}$ is prescribed on $\Gamma_{b}$ (see Figure). Denote by $\mathbb{S}^{d}$ the space of second-order symmetric tensors on $\mathbb{R}^{d}$ and by "." and $\|$.$\| the inner product and the Euclidean norms on \mathbb{R}^{d}$ and $\mathbb{S}^{d}$, respectively. Finally, let $[0, T], T>0$, be the time interval of interest and denote by $v$ the unit outer normal vector to $\Gamma$. Let $x \in \Omega$ and $t \in[0, T]$ be the spacial and the time variables, respectively, and, to simplify the writing, we do not indicate the dependence of the functions on $x$ and $t$. With these assumptions, the classical formulation of the electro-elastic contact problem with adhesion is the following.

Problem P: Find a displacement field $u: \Omega \times(0, T) \longrightarrow \mathbb{R}^{d}$, a stress field $\sigma: \Omega \times(0, T) \longrightarrow \mathbb{S}^{d}$, an electric potential $\varphi: \Omega \times(0, T) \longrightarrow \mathbb{R}$, an electric displacement field $\mathbf{D}: \Omega \times(0, T) \longrightarrow \mathbb{R}^{d}$ and a bonding field $\beta: \Omega \times(0, T) \longrightarrow \mathbb{R}$ such that

$$
\begin{aligned}
& \sigma=\mathcal{F} \varepsilon(u)-\mathcal{E}^{*} E(\varphi) \text { in } \Omega \times(0, T), \\
& \mathbf{D}=\mathcal{B} \mathbf{E}(\varphi)+\mathcal{E} \varepsilon(u) \text { in } \Omega \times(0, T), \\
& \operatorname{Div} \sigma+f_{0}=0 \quad \text { in } \Omega \times(0, T), \\
& \operatorname{div} \mathbf{D}=q_{0} \text { in } \Omega \times(0, T), \\
& u=0 \text { on } \Gamma_{1} \times(0, T), \\
& \sigma v=f_{2} \text { on } \Gamma_{2} \times(0, T), \\
& u_{v} \leq 0, \sigma_{v}+p_{v}\left(\beta, u_{v}\right) \leq 0, u_{v}\left(\sigma_{v}+p_{v}\left(\beta, u_{v}\right)\right)=0 \quad \text { on } \Gamma_{3} \times(0, T), \\
& -\sigma_{\tau}=p_{\tau}\left(\beta, u_{\tau}\right) \text { on } \Gamma_{3} \times(0, T), \\
& \dot{\beta}=H_{a d}\left(\beta, \zeta_{\beta}, R\left(\left|u_{\tau}\right|\right)\right) \text { on } \Gamma_{3} \times(0, T), \\
& \varphi=0 \text { on } \Gamma_{a} \times(0, T), \\
& \mathbf{D} \cdot v=q_{2} \text { on } \Gamma_{b} \times(0, T) \\
& \text { D. } v=0 \text { on } \Gamma_{3} \times(0, T), \\
& \beta(0)=\beta_{0} \text { on } \Gamma_{3} .
\end{aligned}
$$


Now, we provide some comments on equations and conditions (1)-(13). Equations (1) and (2) represent the electro-elastic constitutive law in which $\varepsilon(u)$ denotes the linearized strain tensor, $\mathbf{E}(\varphi)=-\nabla \varphi$ is the electric field, where $\varphi$ is the electric potential, $\mathcal{F}$ is a given nonlinear function, $\mathcal{E}$ represents the piezoelectric operator, $\mathcal{E}^{*}$ is its transpose, $\mathcal{B}$ denotes the electric permittivity operator, and $\mathbf{D}=\left(D_{1}, \ldots, D_{d}\right)$ is the electric displacement vector. Details on the constitutive equations of the form (1) and (2) can be found, for instance, in [4,6]. Next, (3) and (4) are the equilibrium equations for the stress and electric displacement fields, respectively, in which "Div" and "div" denote the divergence operator for tensor and vector valued functions, respectively. Equations (5) and (6) represent the displacement and traction boundary conditions, whereas (10) and (11) represent the electric boundary conditions. We consider the condition (12) due to physical reasons. The evolution of the adhesion field is governed by the differential equation (9). Conditions (7) represent the Signorini contact condition with adhesion where $u_{v}$ is the normal displacement, and $\sigma_{v}$ represents the normal stress. In this paper, $p_{v}$ is a prescribed function which depends on the adhesion and normal displacement.

A usual choice of the function $p_{v}$ is (see, e.g., [11])

$$
p_{v}\left(\beta, u_{v}\right)=c_{\nu} \beta^{2} R_{\nu}\left(u_{v}\right),
$$

where $c_{\nu}$ is a positive constant and $R_{\nu}$ is the truncation operator defined by

$$
R_{v}(s)= \begin{cases}L & \text { if } s<-L \\ -s & \text { if }-L \leq s \leq 0 \\ 0 & \text { if } s>0\end{cases}
$$

Here, $L>0$ is the characteristic length of the bond of the glue, beyond which it does not offer any additional traction.

We assume that the resistance to tangential motion is generated by the glue, in comparison to which the frictional traction can be neglected. A different assumption, taking frictional traction into account, can be found in $[14,17]$. Thus, the tangential traction depends only on the intensity of adhesion, and the tangential displacement,

$$
-\sigma_{\tau}=p_{\tau}\left(\beta, u_{\tau}\right) \quad \text { on } \Gamma_{3} \times(0, T) .
$$

In particular, we may consider the case

$$
p_{\tau}(\beta, r)= \begin{cases}q_{\tau}(\beta) r, & \text { if }\|r\| \leq L_{0}, \\ q_{\tau}(\beta) \frac{L_{0}}{\|r\|} r, & \text { if }\|r\|>L_{0},\end{cases}
$$

where $L_{0}$ is the limit bound length and $q_{\tau}$ is a nonnegative tangential stiffness function. A more general condition can be used, especially if the surface has intrinsic directions, such as grooves, on it. Then, one needs to replace $q_{\tau}$ with a two-dimensional tensor. The theory below can be extended to such cases easily. As in [9], the evolution of the adhesion field is assumed to depend generally on $\beta$ and $u_{\tau}$. We do not impose sign restrictions on the process and, thus, cycles of debonding and rebonding may take place, as a result of imposed periodic forces or displacements. In addition, we include here the possibility that, as the cycles of bonding and debonding go on, there is a decrease in the bonding effectiveness. Therefore, the process is also assumed to depend on the time history of the bonding, which we denote by

$$
\zeta_{\beta}(x, t)=\int_{0}^{t} \beta(x, s) \mathrm{d} s \quad \text { on } \Gamma_{3} \times(0, T) .
$$

The whole process is assumed to be governed by the differential equation,

$$
\dot{\beta}=H_{\mathrm{ad}}\left(\beta, \zeta_{\beta}, R\left(\left|u_{\tau}\right|\right)\right) \quad \text { on } \Gamma_{3} \times(0, T),
$$

where $H_{\text {ad }}$ is a general function discussed below, which vanishes when its first argument vanishes. The function $R: \mathbb{R}_{+} \rightarrow \mathbb{R}_{+}$is a truncation operator and is defined as

$$
R(s)= \begin{cases}s, & \text { if } 0 \leq s \leq L, \\ L, & \text { if } s>L,\end{cases}
$$


where $L>0$ is a characteristic length of the bonds (see, e.g., [17]). We use it in $H_{\text {ad }}$, since usually when the glue is stretched beyond the limit $L$ it does not contribute more to the bond strength. An example of such a function, used in [8], is

$$
H_{\mathrm{ad}}\left(\beta, R\left(\left|u_{\tau}\right|\right)=-\gamma_{\nu} \beta_{+} R\left(\left|u_{\tau}\right|\right)^{2},\right.
$$

where $\gamma_{v}$ is the bonding energy constant and $\gamma_{v} L$ is the maximal tensile normal traction that the adhesive can provide and $\beta_{+}=\max (0, \beta)$. We note that in this case, only debonding is allowed. Another example, in which $H_{\text {ad }}$ depends on all three variables is

$$
H_{\mathrm{ad}}(\beta, \zeta, r)=-\gamma_{\nu} \beta_{+} r^{2}+\frac{\gamma_{\nu} \beta_{+}(1-\beta)_{+}}{1+\zeta^{2}} .
$$

Here, the tangential displacement $r$ causes the debonding, while naturally there is a tendency to rebound. However, the bonding cannot exceed $\beta=1$ and, moreover, the rebonding becomes weaker as the process goes on, which is represented by the factor $1+\zeta^{2}$ in the denominator. Finally, (13) is the initial condition in which $\beta_{0}$ is a given bonding field.

\section{Variational Formulation and Preliminaries}

In this section, we list the assumptions on the data and derive a variational formulation for the contact problem. To this end, we need to introduce some notation and preliminary material. Here and below, $\mathbb{S}^{d}$ represent the space of second-order symmetric tensors on $\mathbb{R}^{d}$. We recall that the inner products and the corresponding norms on $\mathbb{S}^{d}$ and $\mathbb{R}^{d}$ are given by

$$
\begin{aligned}
& u . v=u_{i} \cdot v_{i}, \quad\|v\|=(v \cdot v)^{\frac{1}{2}} \quad \forall u, v \in \mathbb{R}^{d}, \\
& \sigma . \tau=\sigma_{i j} \cdot \tau_{i j}, \quad\|\tau\|=(\tau . \tau)^{\frac{1}{2}} \quad \forall \sigma, \tau \in \mathbb{S}^{d} .
\end{aligned}
$$

Here and elsewhere in this paper, $i, j, k, l$ run from 1 to $d$, summation over repeated index is applied and the index that follows a comma represents the partial derivative with respect to the corresponding component of the spatial variable, e.g., $u_{i, j}=\frac{\partial u_{i}}{\partial x_{j}}$.

Everywhere below, we use the classical notation for $L^{p}$ and Sobolev spaces associated with $\Omega$ and $\Gamma$. Moreover, we use the notation $H, H_{1}$ and $\mathcal{H}, \mathcal{H}_{1}$ for the following spaces:

$$
\begin{aligned}
& H=L^{2}(\Omega)^{d}=\left\{v=\left(v_{i}\right) ; v_{i} \in L^{2}(\Omega)\right\}, \quad \mathcal{H}=\left\{\tau=\left(\tau_{i j}\right) ; \tau_{i j}=\tau_{j i} \in L^{2}(\Omega)\right\}, \\
& H_{1}=H^{1}(\Omega)^{d}=\{v \in H ; \varepsilon(v) \in \mathcal{H}\}, \quad \mathcal{H}_{1}=\{\tau \in \mathcal{H} ; \operatorname{Div} \tau \in H\} .
\end{aligned}
$$

The spaces $H, H_{1}, \mathcal{H}$ and $\mathcal{H}_{1}$ are real Hilbert spaces endowed with the canonical inner products given by

$$
\begin{aligned}
& (u, v)_{H}=\int_{\Omega} u . v \mathrm{~d} x, \quad(u, v)_{H_{1}}=\int_{\Omega} u . v \mathrm{~d} x+\int_{\Omega} \nabla u . \nabla v \mathrm{~d} x, \\
& (\sigma, \tau)_{\mathcal{H}}=\int_{\Omega} \sigma \cdot \tau \mathrm{d} x, \quad(\sigma, \tau)_{\mathcal{H}_{1}}=\int_{\Omega} \sigma . \tau \mathrm{d} x+\int_{\Omega} \operatorname{Div} \sigma \cdot \operatorname{Div} \tau \mathrm{d} x,
\end{aligned}
$$

and the associated norms $\|\cdot\|_{H},\|\cdot\|_{H_{1}},\|\cdot\|_{\mathcal{H}}$ and $\|\cdot\|_{\mathcal{H}_{1}}$ respectively.

Here and below, we use the notation

$$
\begin{aligned}
& \nabla v=\left(v_{i, j}\right), \quad \varepsilon(v)=\left(\varepsilon_{i j}(v)\right), \quad \varepsilon_{i j}(v)=\frac{1}{2}\left(v_{i, j}+v_{j, i}\right) \quad \forall v \in H_{1}, \\
& \operatorname{Div} \tau=\left(\tau_{i j, j}\right) \quad \forall \tau \in \mathcal{H}_{1} .
\end{aligned}
$$

For every element $v \in H_{1}$, we also write $v$ for the trace of $v$ on $\Gamma$ and we denote by $v_{v}$ and $v_{\tau}$ the normal and tangential components of $v$ on $\Gamma$ given by $v_{\nu}=v \cdot v, v_{\tau}=v-v_{\nu} v$. Let us now consider the closed subspace 
of $H^{1}(\Omega)^{d}$, defined by $V=\left\{v \in H_{1} ; v=0\right.$ on $\left.\Gamma_{1}\right\}$. Since meas $\left(\Gamma_{1}\right)>0$, the following Korn's inequality holds:

$$
\|\varepsilon(v)\|_{\mathcal{H}} \geq c_{K}\|v\|_{H_{1}} \quad \forall v \in V
$$

where $c_{K}>0$ is a constant which depends only on $\Omega$ and $\Gamma_{1}$. Over the space $V$, we consider the inner product given by

$$
(u, v)_{V}=(\varepsilon(u), \varepsilon(v))_{\mathcal{H}}
$$

and let $\|\cdot\|_{V}$ be the associated norm. It follows from Korn's inequality (18) that $\|\cdot\|_{H_{1}}$ and $\|\cdot\|_{V}$ are equivalent norms on $V$ and, therefore, $\left(V,\|\cdot\|_{V}\right)$ is a real Hilbert space. Moreover, by the Sobolev trace theorem, (18) and (19), there exists a constant $c_{0}$ depending only on the domain $\Omega, \Gamma_{1}$ and $\Gamma_{3}$ such that

$$
\|v\|_{L^{2}\left(\Gamma_{3}\right)^{d}} \leq c_{0}\|v\|_{V} \quad \forall v \in V .
$$

We also introduce the following spaces:

$$
\begin{aligned}
& W=\left\{\psi \in H^{1}(\Omega) \text { such that } \psi=0 \text { on } \Gamma_{a}\right\}, \\
& \mathcal{W}_{1}=\left\{D=\left(D_{i}\right) \text { such that } D_{i} \in L^{2}(\Omega), \quad D_{i, i} \in L^{2}(\Omega)\right\} .
\end{aligned}
$$

Since meas $\left(\Gamma_{a}\right)>0$, the following Friedrichs-Poincaré inequality holds:

$$
\|\nabla \psi\|_{L^{2}(\Omega)^{d}} \geq c_{F}\|\psi\|_{H^{1}(\Omega)} \quad \forall \psi \in W,
$$

where $c_{F}>0$ is a constant which depends only on $\Omega, \Gamma_{a}$ and $\nabla \psi=\left(\psi_{, i}\right)$. Over the space $W$, we consider the inner product given by

$$
(\varphi, \psi)_{W}=\int_{\Omega} \nabla \varphi \cdot \nabla \psi \mathrm{d} x
$$

and let $\|\cdot\|_{W}$ be the associated norm. It follows from (21) that $\|\cdot\|_{H^{1}(\Omega)}$ and $\|\cdot\|_{W}$ are equivalent norms on $W$ and therefore $\left(W,\|\cdot\|_{W}\right)$ is a real Hilbert space. Moreover, by the Sobolev trace theorem, there exists a constant $c_{0}$, depending only on $\Omega, \Gamma_{a}$ and $\Gamma_{3}$, such that

$$
\|\zeta\|_{L^{2}\left(\Gamma_{3}\right)} \leq \tilde{c}_{0}\|\zeta\|_{W} \quad \forall \zeta \in W .
$$

The space $\mathcal{W}_{1}$ is real Hilbert space with the inner product

$$
(\mathbf{D}, \mathbf{E})_{\mathcal{W}_{1}}=\int_{\Omega} \mathbf{D} . \mathbf{E} \mathrm{d} x+\int_{\Omega} \operatorname{div} \mathbf{D} \cdot \operatorname{div} \mathbf{E} \mathrm{d} x,
$$

where $\operatorname{div} \mathbf{D}=\left(D_{i, i}\right)$ and the associated norm $\|\cdot\| \mathcal{W}_{1}$.

For every real Hilbert space $X$, we use the classical notation for the spaces $L^{p}(0, T ; X)$ and $W^{k, p}(0, T ; X), 1 \leq p \leq \infty, k \geq 1$ and we also introduce the set

$$
\mathcal{Q}=\left\{\theta \in L^{\infty}\left(0, T ; L^{2}\left(\Gamma_{3}\right)\right) \text { such that } 0 \leq \theta(t) \leq 1 \quad \forall t \in[0, T] \text {, a.e. on } \Gamma_{3}\right\} .
$$

Finally, if $X_{1}$ and $X_{2}$ are two Hilbert spaces endowed with the inner products $(., .)_{X_{1}}$ and $(.,)_{X_{2}}$, the associated norms $\|\cdot\|_{X_{1}}$ and $\|\cdot\|_{X_{2}}$, respectively, we denote by $X_{1} \times X_{2}$ the product space together with the canonical inner product $(.,)_{X_{1} \times X_{2}}$ and the associated norm $\|.\|_{X_{1} \times X_{2}}$. In the study of the Problem $\mathbf{P}$, we consider the following assumptions:

We assume that the elasticity operator $\mathcal{F}: \Omega \times \mathbb{S}^{d} \rightarrow \mathbb{S}^{d}$ satisfies:

(a) There exists $L_{\mathcal{F}}>0$ such that $\left\|\mathcal{F}\left(x, \varepsilon_{1}\right)-\mathcal{F}\left(x, \varepsilon_{2}\right)\right\| \leq L_{\mathcal{F}}\left\|\varepsilon_{1}-\varepsilon_{2}\right\|$

$\forall \varepsilon_{1}, \varepsilon_{2} \in \mathbb{S}^{d}$, a.e. $x \in \Omega$,

(b) there exists $m_{\mathcal{F}}>0$ such that

$$
\begin{aligned}
& \left(\mathcal{F}\left(x, \varepsilon_{1}\right)-\mathcal{F}\left(x, \varepsilon_{2}\right)\right) .\left(\varepsilon_{1}-\varepsilon_{2}\right) \geq m_{\mathcal{F}}\left\|\varepsilon_{1}-\varepsilon_{2}\right\|^{2} \\
& \forall \varepsilon_{1}, \varepsilon_{2} \in \mathbb{S}^{d}, \quad \text { a.e. } x \in \Omega,
\end{aligned}
$$

(c) the map $x \mapsto \mathcal{F}(x, \varepsilon)$ is Lebesgue measurable in $\Omega$

$\forall \varepsilon \in \mathbb{S}^{d}$, a.e. $x \in \Omega$,

(d) the map $x \mapsto \mathcal{F}(x, 0) \in \mathcal{H}$. 
The piezoelectric tensor $\mathcal{E}: \Omega \times \mathbb{S}^{d} \rightarrow \mathbb{R}^{d}$ satisfies:

$$
\left\{\begin{array}{l}
\text { (a) } \mathcal{E}(x, \tau)=\left(e_{i j k}(x) \tau_{j k}\right) \quad \forall \tau=\left(\tau_{i j}\right) \in \mathbb{S}^{d}, \quad \text { a.e. } x \in \Omega \\
\text { (b) } e_{i j k}=e_{i k j} \in L^{\infty}(\Omega)
\end{array}\right.
$$

The permittivity operator $\mathcal{B}: \Omega \times \mathbb{R}^{d} \rightarrow \mathbb{R}^{d}$ verifies:

$$
\left\{\begin{array}{l}
\text { (a) } \mathcal{B}(x, \mathbf{E})=\left(b_{i j}(x) E_{j}\right) \quad \forall \mathbf{E}=\left(E_{i}\right) \in \mathbb{R}^{d}, \quad \text { a.e. } x \in \Omega, \\
\text { (b) } b_{i j}=b_{j i} \in L^{\infty}(\Omega) \text { for } i, j=1, \ldots, d, \\
\text { (c) there exists } m_{\mathcal{B}}>0 \text { such that } b_{i j}(x) E_{i} E_{j} \geq m_{\mathcal{B}}\|\mathbf{E}\|^{2} \\
\forall \mathbf{E}=\left(E_{i}\right) \in \mathbb{R}^{d}, \quad \text { a.e. } x \in \Omega
\end{array}\right.
$$

Recall also that the transposed operator $\mathcal{E}^{*}$ is given by $\mathcal{E}^{*}=\left(e_{i j k}^{*}\right)$ where $e_{i j k}^{*}=e_{k i j}$ and the following equality holds

$$
\mathcal{E} \sigma \cdot v=\sigma \mathcal{E}^{*} v, \quad \forall \sigma \in \mathbb{S}^{d}, v \in \mathbb{R}^{d} .
$$

The normal function $p_{v}: \Gamma_{3} \times \mathbb{R} \times \mathbb{R} \longrightarrow \mathbb{R}_{+}$satisfies:

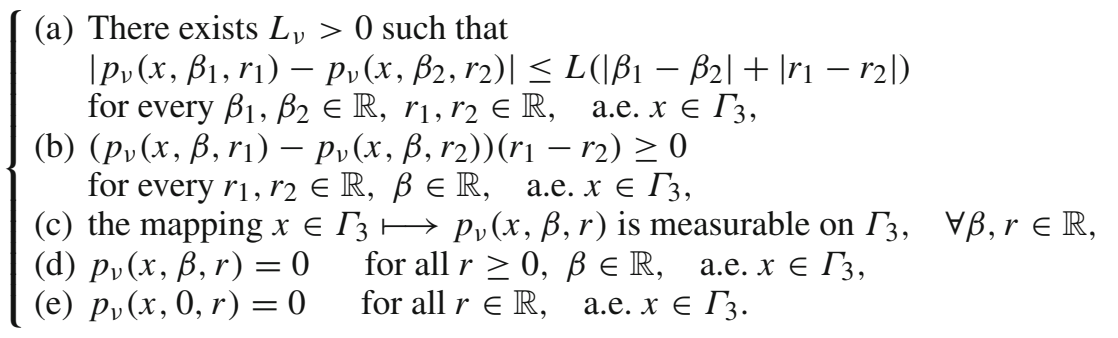

The tangential function $p_{\tau}: \Gamma_{3} \times \mathbb{R} \times \mathbb{R}^{d} \longrightarrow \mathbb{R}^{d}$ satisfies:

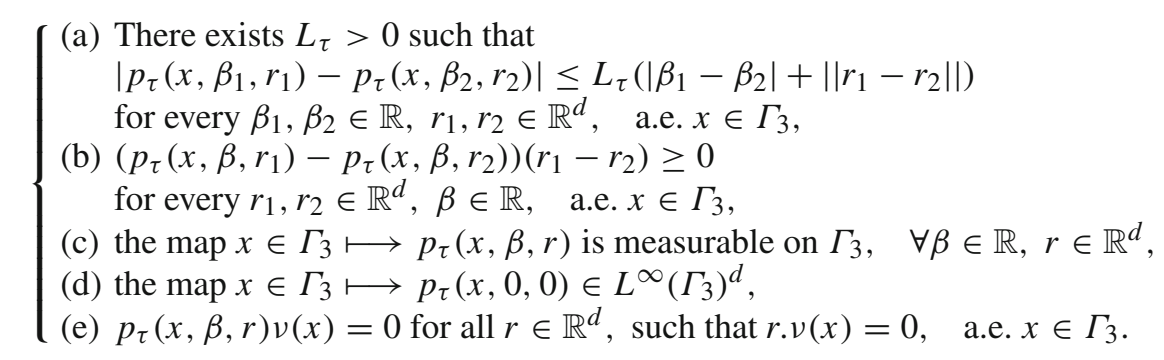

Next, the adhesion rate function $H_{\mathrm{ad}}: \Gamma_{3} \times \mathbb{R} \times \mathbb{R} \times[-L, L] \rightarrow \mathbb{R}$ satisfies:

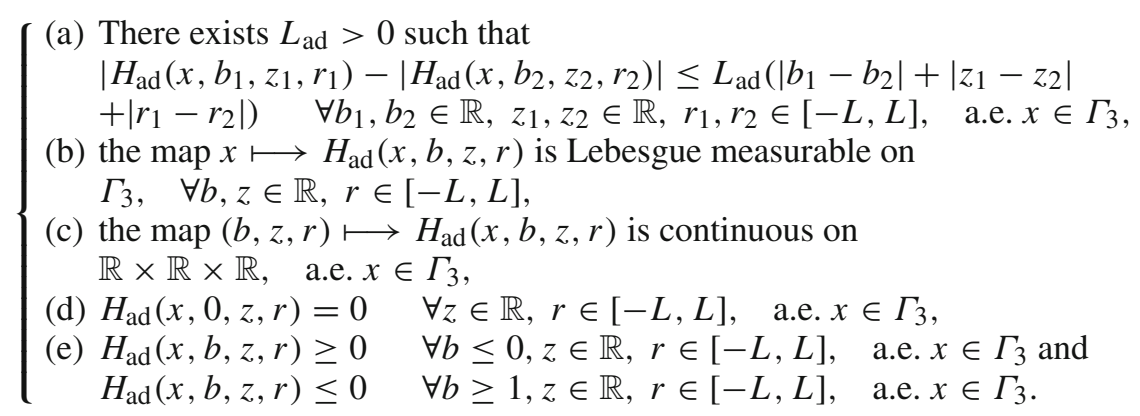

We remark that if $\beta \in L^{2}\left(\Gamma_{3}\right), z \in L^{2}\left(\Gamma_{3}\right)$ and $r: \Gamma_{3} \rightarrow \mathbb{R}$ is a measurable function, then the conditions (29) imply that $x \mapsto H_{a d}(x, \beta(x), z(x), \operatorname{Rr}(x)) \in L^{2}\left(\Gamma_{3}\right)$.

The following regularity is assumed on the density of volume forces, traction, volume electric charges and surface electric charges:

$$
\left\{\begin{array}{l}
f_{0} \in W^{1, \infty}([0, T] ; H), \quad f_{2} \in W^{1, \infty}\left([0, T] ;\left[L^{2}\left(\Gamma_{2}\right)\right]^{d}\right) \\
q_{0} \in W^{1, \infty}\left([0, T] ; L^{2}(\Omega)\right), \quad q_{2} \in W^{1, \infty}\left([0, T] ; L^{2}\left(\Gamma_{b}\right)\right) .
\end{array}\right.
$$


Finally, the initial displacements and adhesion conditions satisfies:

$$
\beta_{0} \in L^{2}\left(\Gamma_{3}\right), \quad 0 \leq \beta_{0}(x) \leq 1 \text { a.e. } x \in \Gamma_{3} .
$$

Using the Riesz theorem, we define the linear mappings $f:[0, T] \longrightarrow V$ and $q:[0, T] \longrightarrow W$ as follows:

$$
\begin{aligned}
& (f(t), v)=\int_{\Omega} f_{0}(t) \cdot v \mathrm{~d} x+\int_{\Gamma_{2}} f_{2}(t) \cdot v \mathrm{~d} a \quad \forall v \in V, \\
& (q(t), \psi)=\int_{\Omega} q_{0}(t) \cdot \psi \mathrm{d} x-\int_{\Gamma_{b}} q_{2}(t) . \psi \mathrm{d} a \quad \forall \psi \in W .
\end{aligned}
$$

For the Signorini Problem, we use the convex subset of admissible displacements given by

$$
U_{\text {ad }}=\left\{v \in H_{1} \text { such that } v=0 \text { on } \Gamma_{1}, v_{\nu} \leq 0 \text { on } \Gamma_{1}\right\} .
$$

We define the functional $j: L^{2}\left(\Gamma_{3}\right) \times V \times V \rightarrow \mathbb{R}$ by

$$
j(\beta, u, v)=\int_{\Gamma_{3}} p_{v}\left(\beta, u_{v}\right) \cdot v_{v} \mathrm{~d} a+\int_{\Gamma_{3}} p_{\tau}\left(\beta, u_{\tau}\right) \cdot v_{\tau} \mathrm{d} a \quad \forall \beta \in L^{2}\left(\Gamma_{3}\right), \forall u, v \in V .
$$

By a standard procedure based on Green's formula, we derive the following variational formulation of the mechanical Problem P.

Problem VP: Find a displacement field $u:[0, T] \longrightarrow V$, an electric potential $\varphi:[0, T] \longrightarrow W$ and a bonding field $\beta:[0, T] \longrightarrow L^{2}\left(\Gamma_{3}\right)$ such that

$$
\begin{aligned}
& \left\{\begin{array}{c}
u(t) \in U_{\mathrm{ad}}, \quad(\mathcal{F} \varepsilon(u(t)), \varepsilon(v-u(t)))_{\mathcal{H}}+\left(\mathcal{E}^{*} \nabla \varphi(t), \varepsilon(v-u(t))\right)_{\mathcal{H}} \\
\quad+j(\beta(t), u(t), v-u(t)) \geq(f(t), v-u(t))_{V} \quad \forall v \in U_{\mathrm{ad}}, t \in(0, T),
\end{array}\right. \\
& (\mathcal{B} \nabla \varphi(t), \nabla \psi)_{L^{2}(\Omega)^{d}}-(\mathcal{E} \varepsilon(u(t)), \nabla \psi)_{L^{2}(\Omega)^{d}}=(q(t), \psi)_{W} \quad \forall \psi \in W, t \in(0, T), \\
& \dot{\beta}(t)=H_{\mathrm{ad}}\left(\beta(t), \zeta_{\beta}(t), R\left(\left|u_{\tau}(t)\right|\right)\right) \quad \text { a.e. } t \in(0, T), \\
& \beta(0)=\beta_{0} .
\end{aligned}
$$

\section{Existence and Uniqueness Result}

Our main existence and uniqueness result is the following.

Theorem 4.1 Assume that (23)-(31) hold. Then there exists a unique solution $\{u, \varphi, \beta\}$ to Problem VP with the following regularity:

$$
\begin{aligned}
& u \in W^{1, \infty}(0, T ; V), \\
& \varphi \in W^{1, \infty}(0, T ; W), \\
& \beta \in W^{1, \infty}\left(0, T ; L^{2}\left(\Gamma_{3}\right)\right) \cap \mathcal{Q} .
\end{aligned}
$$

We conclude that under assumptions (23)-(31) Problem $\mathbf{P}$ has a unique weak solution satisfying (38)(40). To precise the regularity of a weak solution, we note that the constitutive relations (1), (2) and (3), (4), the assumptions ( 23),(26) and the regularities (38),(39) show that $\sigma \in W^{1, \infty}(0, T ; \mathcal{H}), \mathbf{D} \in$ $W^{1, \infty}\left(0, T ; L^{2}(\Omega)^{d}\right)$; moreover, equalities (32) and (33) combined with the definitions of $f, q$ and functional $j$ yield

$$
\operatorname{Div} \sigma(t)+f_{0}(t)=0, \quad \operatorname{div} \mathbf{D}(t)=q_{0}(t) \quad \forall t \in(0, T) .
$$

It follows now from the regularities (24) and the transposed operator $\mathcal{E}^{*}$ given by assumption (26) that $\operatorname{Div} \sigma \in$ $W^{1, \infty}\left(0, T ; L^{2}(\Omega)^{d}\right)$ and $\operatorname{div} \mathbf{D} \in W^{1, \infty}\left(0, T ; L^{2}(\Omega)\right)$, which shows that

$$
\begin{aligned}
& \sigma \in W^{1, \infty}\left(0, T ; \mathcal{H}_{1}\right), \\
& \mathbf{D} \in W^{1, \infty}\left(0, T ; \mathcal{W}_{1}\right) .
\end{aligned}
$$


We conclude that the weak solution $(u, \sigma, \varphi, \mathbf{D}, \beta)$ of the electro-elastic contact Problem $\mathbf{P}$ has the regularity (38), (41), (39), (42) and (40).

In the proofs of the Theorem 4.1, we use a number of inequalities involving the functional $j$ that we present in what follows. Below in this section, $\beta, \beta_{1}, \beta_{2}$ denote elements of $L^{2}\left(\Gamma_{3}\right)$ such that $0 \leq \beta, \beta_{1}, \beta_{2} \leq 1$ a.e. on $\Gamma_{3}, u_{1}, u_{2}$ and $v$ are elements of $V$, and $c>0$ is a generic constant which may depend of $\Omega, \Gamma_{1}, \Gamma_{3}, p_{v}, p_{\tau}$ and $L$.

First, we notice that the function $j$ is linear with respect to the last argument and therefore

$$
j(\beta, u,-v)=-j(\beta, u, v) .
$$

Next, we use (33), the properties of the truncation operator $R$ as well as assumptions (27) and (28) on the functions $p_{v}, p_{\tau}$. After some calculus, we find

$$
\begin{aligned}
& j\left(\beta, u_{1}, u_{2}-u_{1}\right)+j\left(\beta, u_{2}, u_{1}-u_{2}\right)=\int_{\Gamma_{3}}\left[\left(p_{v}\left(x, \beta, u_{1}\right)-p_{v}\left(x, \beta, u_{2}\right)\right)\left(u_{2}-u_{1}\right)\right. \\
& \left.\quad+\left(p_{\tau}\left(x, \beta, u_{1}\right)-p_{\tau}\left(x, \beta, u_{2}\right)\right)\left(u_{2}-u_{1}\right)\right] \mathrm{d} a
\end{aligned}
$$

and obtain

$$
j\left(\beta, u_{1}, u_{2}-u_{1}\right)+j\left(\beta, u_{2}, u_{1}-u_{2}\right) \leq 0
$$

With similar computations, based on the Lipschitz continuity of $R, p_{v}$ and $p_{\tau}$, we show that the following inequality also holds:

$$
\left|j\left(\beta, u_{1}, v\right)-j\left(\beta, u_{2}, v\right)\right| \leq c\left\|u_{1}-u_{2}\right\|_{V}\|v\|_{V} .
$$

Also, we take $u_{1}=v$ and $u_{2}=0$ in (45) to obtain

$$
j(\beta, v, v) \geq 0 .
$$

\section{Proof of Theorem 4.1}

The proof of Theorem 4.1 will be carried out in several steps. We denote by $c$ a generic positive constant, which may depend on $\Omega, \Gamma_{1}, \Gamma_{3}, \mathcal{F}, \mathcal{B}, \mathcal{E}$ and $T$, but does not depend on $t$ or on the rest of the input data and whose value may change from place to place. Moreover for the sake of simplicity, we suppress in what follows the explicit dependence on various functions on $x \in \Omega \cup \Gamma_{3}$. $\mathcal{L}$ denotes the closed set of the space $C\left([0, T] ; L^{2}\left(\Gamma_{3}\right)\right)$ defined by

$$
\mathcal{L}=\left\{\beta \in C\left([0, T] ; L^{2}\left(\Gamma_{3}\right)\right) \cap \mathcal{Q} \backslash \beta(0)=\beta_{0}\right\},
$$

and let $\beta \in \mathcal{L}$ be a given function. First, we consider the following intermediaries problems:

Problem VP $\mathbf{P}^{\beta}:$ Find a displacement field $u_{\beta}:[0, T] \longrightarrow V$ and an electric potential field $\varphi_{\beta}:[0, T] \longrightarrow W$ such that

$$
\begin{aligned}
& \left\{\begin{array}{l}
\forall u_{\beta} \in U_{\mathrm{ad}}\left(\mathcal{F} \varepsilon\left(u_{\beta}(t)\right), \varepsilon\left(v-u_{\beta}(t)\right)\right)_{\mathcal{H}}+\left(\mathcal{E}^{*} \nabla \varphi_{\beta}(t), \varepsilon\left(v-u_{\beta}(t)\right)\right)_{\mathcal{H}} \\
+j\left(\beta(t), u_{\beta}(t), v-u_{\beta}(t)\right) \geq\left(f(t), v-u_{\beta}(t)\right)_{V}, \quad \forall v \in U_{\mathrm{ad}}, t \in(0, T),
\end{array}\right. \\
& \left(\mathcal{B} \nabla \varphi_{\beta}(t), \nabla \psi\right)_{L^{2}(\Omega)^{d}}-\left(\mathcal{E} \varepsilon\left(u_{\beta}(t)\right), \nabla \psi\right)_{L^{2}(\Omega)^{d}}=(q(t), \psi)_{W} \quad \forall \psi \in W, t \in(0, T) .
\end{aligned}
$$

To study the variational Problem VP ${ }^{\beta}$, we consider the product space $X=V \times W$ endowed with the inner product

$$
(x, y)=(u, v)_{V}+(\varphi, \psi)_{W}, \quad \forall x=(u, \varphi), y=(v, \psi) \in X,
$$


and the associated norm $\|\cdot\|_{X}$. We also introduce the set $K \subset X$ and the function $A_{\beta}:[0, T] \times X \rightarrow X, \tilde{f}$ : $[0, T] \rightarrow X$, defined by

$$
\begin{aligned}
& K=U_{\mathrm{ad}} \times W \\
& \quad\left(A_{\beta}(t) x, y\right)_{X}=(\mathcal{F} \varepsilon(u(t)), \varepsilon(v))_{\mathcal{H}}+(\mathcal{B} \nabla \varphi(t), \nabla \psi)_{L^{2}(\Omega)^{d}} \\
& \quad+\left(\mathcal{E}^{*} \nabla \varphi(t), \varepsilon(v)\right)_{\mathcal{H}}-(\mathcal{E} \varepsilon(u(t)), \nabla \psi)_{L^{2}(\Omega) d} \\
& +j(\beta(t), u(t), v), \quad \forall x=(u, \varphi), y=(v, \psi) \in X, t \in[0, T], \\
& \tilde{f}(t)=(f(t), q(t)), \quad \forall t \in[0, T] .
\end{aligned}
$$

We start with the following equivalence result

Lemma 5.1 The couple $\left(u_{\beta}, \varphi_{\beta}\right):[0, T] \rightarrow V \times W$ is a solution to Problem $\mathbf{V P}^{\beta}$ if and only if $x_{\beta}:[0, T] \rightarrow$ $X$ satisfies

$$
x_{\beta}(t) \in K, \quad\left(A_{\beta}(t) x_{\beta}(t), y-x_{\beta}(t)\right)_{X} \geq\left(\tilde{f}(t), y-x_{\beta}(t)\right)_{X} \quad \forall y \in K \forall t \in[0, T] .
$$

Proof Let $x_{\beta}(t)=\left(u_{\beta}, \varphi_{\beta}\right):[0, T] \rightarrow V \times W$ be a solution to Problem $\mathbf{V P}^{\beta}$. Let $y=(v, \psi) \in K$ and let $t \in[0, T]$. We use the test function $\psi-\varphi_{\beta}(t)$ in Equality (49), add the corresponding inequality to (48) and use (50)-(54) to obtain Inequality (55). Conversely, assume that $x_{\beta}=\left(u_{\beta}, \varphi_{\beta}\right):[0, T] \rightarrow X$ satisfies Inequality (55) and let $t \in[0, T]$. For any $v \in U_{\text {ad }}$, we take $y=\left(v, \varphi_{\beta}(t)\right)$ in Equality (55) to obtain Inequality (48). Then, for any $\psi \in W$, we take successively $y=\left(u_{\beta}, \varphi_{\beta}+\psi\right)$ and $y=\left(u_{\beta}, \varphi_{\beta}-\psi\right)$ in Equality (55) to obtain (49).

We use now Lemma 5.1 to obtain the following existence and uniqueness result.

Lemma 5.2 Problem VP $\mathbf{P}^{\beta}$ has a unique solution $\left(u_{\beta}, \varphi_{\beta}\right) \in C([0, T] ; V \times W)$.

Proof Let $t \in[0, T]$. We use (23)-(26) and (34) to see that $A_{\beta}(t)$ is a strongly monotone Lipschitz continuous operator on $X$; since $K$ is a nonempty closed convex set of $X$, by a standard result on elliptic variational inequalities, it follows that there exists a unique element $x_{\beta}(t)=\left(u_{\beta}, \varphi_{\beta}\right) \in X$ which satisfies Inequality (55). For $t_{1}, t_{2} \in[0, T]$, an argument based on (23) and (46) shows that

$$
\left\|x_{\beta}\left(t_{1}\right)-x_{\beta}\left(t_{2}\right)\right\|_{X} \leq c\left(\left\|\beta\left(t_{1}\right)-\beta\left(t_{2}\right)\right\|_{L^{2}\left(\Gamma_{3}\right)}+\left\|\tilde{f}\left(t_{2}\right)-\tilde{f}\left(t_{1}\right)\right\|_{X}\right) .
$$

Keeping in mind that $\tilde{f} \in W^{1, \infty}(0, T ; X)$ and that $\beta \in W^{1, \infty}\left(0, T ; L^{2}\left(\Gamma_{3}\right)\right)$, it follows now from Inequality (56) that the mapping $t \mapsto x_{\beta}:[0, T] \rightarrow X$ is continuous. The existence and uniqueness part in Lemma 5.2 is now a consequence of Lemma 5.1.

In the second step we use the displacement field $u_{\beta}$ obtained in Lemma 5.2, denote by $u_{\beta \nu}, u_{\beta \tau}$ its normal and tangential components and we consider the following initial value problem.

Problem $\mathbf{V P}{ }_{\beta}^{\theta}$ : Find a bonding field $\theta_{\beta}:[0, T] \longrightarrow L^{2}\left(\Gamma_{3}\right)$ such that

$$
\begin{aligned}
& \dot{\theta}_{\beta}(t)=H_{\mathrm{ad}}\left(\theta_{\beta}(t), \zeta_{\theta_{\beta}}(t), R\left(\left|u_{\beta \tau}(t)\right|\right)\right) \quad \text { on } \Gamma_{3} \times(0, T), \\
& \theta_{\beta}(0)=\beta_{0} .
\end{aligned}
$$

We have:

Lemma 5.3 There exists a unique solution $\theta_{\beta}$ to Problem $\mathbf{V P}_{\beta}^{\theta}$ such that

$$
\theta_{\beta} \in W^{1, \infty}\left(0, T ; L^{2}\left(\Gamma_{3}\right)\right) \cap \mathcal{Q} .
$$

Proof Let $\zeta \in L^{\infty}\left(0, T ; L^{2}\left(\Gamma_{3}\right)\right)$ and let the mapping $F_{\beta}:[0, T] \times L^{2}\left(\Gamma_{3}\right) \rightarrow L^{2}\left(\Gamma_{3}\right)$ defined by

$$
F_{\beta}(t, \theta)=H_{\mathrm{ad}}\left(\theta_{\beta}(t), \zeta_{\theta_{\beta}}(t), R\left(\left|u_{\beta \tau}(t)\right|\right)\right)
$$

for all $t \in[0, T]$ and $\theta \in L^{2}\left(\Gamma_{3}\right)$. It follows from the properties of the truncation operator $R$ that $F_{\beta}$ is Lipschitz continuous with respect to the second argument, uniformly in time. Moreover, for any $\theta \in L^{2}\left(\Gamma_{3}\right)$, 
the mapping $t \mapsto F_{\beta}(t, \theta)$ belongs to $L^{\infty}\left(0, T ; L^{2}\left(\Gamma_{3}\right)\right)$. Using now a version of Cauchy-Lipschitz theorem, we obtain the existence of a unique function $\theta_{\beta \zeta} \in W^{1, \infty}\left(0, T ; L^{2}\left(\Gamma_{3}\right)\right)$ such that

$$
\begin{aligned}
& \dot{\theta}_{\beta \zeta}(t)=H_{\mathrm{ad}}\left(\theta_{\beta \zeta}(t), \zeta_{\theta}(t), R\left(\left|u_{\beta \tau}(t)\right|\right)\right) \\
& \theta_{\beta \zeta}(0)=\beta_{0}
\end{aligned}
$$

We note that the restriction $0 \leq \beta \leq 1$ is implicitly included in the variational Problem VP. Indeed, (36) and (37) guarantee that $\beta(t) \leq \beta_{0}$ and, therefore, assumption (31) shows that $\beta \leq 1$ for $t \geq 0$, a.e. on $\Gamma_{3}$. On the other hand, if $\beta\left(t_{0}\right)=0$ at $t=t_{0}$, then it follows from (36) and (37) that $\dot{\beta}(t)=0$ for all $t \geq t_{0}$ and, therefore, $\beta(t)=0$ for all $t \geq 0$, a.e. on $\Gamma_{3}$. We conclude that $0 \leq \beta(t) \leq 1$ for all $t \in[0, T]$, a.e. on $\Gamma_{3}$. Therefore, from the definition of the set $\mathcal{Q}$, we find that $\theta_{\beta \zeta} \in \mathcal{Q}$, which concludes the proof of lemma.

It follows from Lemma 5.3 that for all $\beta \in \mathcal{L}$ and $\zeta \in L^{\infty}\left(0, T ; L^{2}\left(\Gamma_{3}\right)\right)$ the solution $\theta_{\beta \zeta}$ to Problem $\mathbf{V P}{ }_{\beta}^{\theta}$ belongs to $\mathcal{L}$ (see the definition of $L$ ).

Consider now the operator $\Lambda: L^{\infty}\left(0, T ; L^{2}\left(\Gamma_{3}\right)\right) \rightarrow L^{\infty}\left(0, T ; L^{2}\left(\Gamma_{3}\right)\right)$ given by

$$
\Lambda \beta(t)=\theta_{\beta}
$$

and we prove that it has a unique fixed point.

Lemma 5.4 There exists a unique element $\beta^{*} \in L^{\infty}\left(0, T ; L^{2}\left(\Gamma_{3}\right)\right)$ such that $\Lambda \beta^{*}=\beta^{*}$.

Proof Suppose that $\beta_{i}$ are two functions in $\mathcal{L}$, and denote by $\left(u_{i}, \varphi_{i}\right), \theta_{i}$ the functions obtained in Lemmas 5.2 and 5.3, respectively, for $\beta=\beta_{i}, i=1,2$. Let $t \in[0, T]$. We use arguments similar to those used in the proof of (56) to deduce that

$$
\left\|u_{1}(t)-u_{2}(t)\right\|_{V}+\left\|\varphi_{1}(t)-\varphi_{2}(t)\right\|_{W} \leq c\left\|\beta_{1}(t)-\beta_{2}(t)\right\|_{L^{2}\left(\Gamma_{3}\right)},
$$

which implies that

$$
\left\|u_{1}(t)-u_{2}(t)\right\|_{V} \leq c\left\|\beta_{1}(t)-\beta_{2}(t)\right\|_{L^{2}\left(\Gamma_{3}\right)} ;
$$

on the other hand, it follows from (57), (58) for $i=1,2$. Let $t \in[0, T]$, we have

$$
\theta_{i}(t)=\beta_{0}+\int_{0}^{t} H_{\mathrm{ad}}\left(\theta_{i}(s), \zeta_{i}(s), R\left(\left|u_{i \tau}(r)\right|\right)\right) \mathrm{d} s
$$

and, using assumption (29a), we find

$$
\begin{aligned}
& \left\|\theta_{1}(t)-\theta_{2}(t)\right\|_{L^{2}\left(\Gamma_{3}\right)} \leq L_{\mathrm{ad}} \int_{0}^{t}\left|\theta_{1}(s)-\theta_{2}(s)\right| \mathrm{d} s+L_{\mathrm{ad}} \int_{0}^{t}\left|\zeta_{1}(s)-\zeta_{2}(s)\right| \mathrm{d} s \\
& \quad+L_{\mathrm{ad}} \int_{0}^{t}\left\|u_{1}(s)-u_{2}(s)\right\|_{V} \mathrm{~d} s .
\end{aligned}
$$

Applying Gronwall's inequality yields

$$
\left\|\theta_{1}(t)-\theta_{2}(t)\right\|_{L^{2}\left(\Gamma_{3}\right)} \leq c \int_{0}^{t}\left\|\zeta_{1}(s)-\zeta_{2}(s)\right\| \mathrm{d} s+c \int_{0}^{t}\left\|u_{1}(s)-u_{2}(s)\right\|_{V} \mathrm{~d} s
$$

and, using (15), we obtain

$$
\left\|\zeta_{1}(s)-\zeta_{2}(s)\right\| \leq c\left\|\beta_{1}(t)-\beta_{2}(t)\right\|_{L^{2}\left(\Gamma_{3}\right)}
$$


therefore, we obtain

$$
\left\|\theta_{1}(t)-\theta_{2}(t)\right\|_{L^{2}\left(\Gamma_{3}\right)} \leq c \int_{0}^{t}\left\|u_{1}(s)-u_{2}(s)\right\|_{V} \mathrm{~d} s+c \int_{0}^{s}\left\|\beta_{1}(s)-\beta_{2}(s)\right\|_{L^{2}\left(\Gamma_{3}\right)} \mathrm{d} s .
$$

From (61) and (62), we find

$$
\left\|\Lambda \beta_{1}(t)-\Lambda \beta_{2}(t)\right\|_{L^{2}\left(\Gamma_{3}\right)} \leq c \int_{0}^{s}\left\|\beta_{1}(s)-\beta_{2}(s)\right\|_{L^{2}\left(\Gamma_{3}\right)} \mathrm{d} s \quad \forall t \in[0, T] .
$$

Reiterating this inequality $n$ times yields

$$
\left\|\Lambda^{n} \beta_{1}-\Lambda^{n} \beta_{2}\right\|_{L^{\infty}\left(0, T ; L^{2}\left(\Gamma_{3}\right)\right)} \leq \frac{(c T)^{2 n}}{(2 n) !}\left\|\beta_{1}-\beta_{2}\right\|_{L^{\infty}\left(0, T ; L^{2}\left(\Gamma_{3}\right)\right)}
$$

which implies that for a sufficiently large $n$, the mapping $\Lambda^{n}$ is a contraction in the Banach space $L^{\infty}\left(0, T ; L^{2}\left(\Gamma_{3}\right)\right)$. Therefore, there exists a unique $\beta^{*} \in L^{\infty}\left(0, T ; L^{2}\left(\Gamma_{3}\right)\right)$ such that $\Lambda^{n} \beta^{*}=\beta^{*}$ and, moreover, $\beta^{*}$ is the unique fixed point of $\Lambda$.

Let $\theta_{\beta}=\theta_{\beta^{*}}$ be the solution of Problem (57), (58) for $\beta=\beta^{*}$. This concludes the existence part of Lemma 5.3. The uniqueness part follows from the uniqueness of the fixed point of the operator $\Lambda$ given by (61).

Now, we have all the ingredients to prove the Theorem 4.1.

Existence: Let $\beta^{*} \in \mathcal{L}$ be the fixed point of the operator $\Lambda$ and let $u_{\eta^{*}}, \varphi_{\eta^{*}}$ the solutions to the Problems VP for $\beta=\beta^{*}$, i.e., $u^{*}=u_{\beta^{*}}$ and $\varphi^{*}=\varphi_{\beta^{*}}$. Since $\theta_{\beta^{*}}=\beta^{*}$, we conclude by (48), (49), (57) and (58) that $\left(u^{*}, \varphi^{*}, \beta^{*}\right)$ is a solution to Problems VP and, moreover, $\beta^{*}$ satisfies the regularity (40). Also, since $\beta^{*}=\theta_{\beta^{*}} \in W^{1, \infty}\left(0, T ; L^{2}\left(\Gamma_{3}\right)\right)$, Inequality (56) implies that the function $x^{*}=\left(u^{*}, \varphi^{*}\right)$ belongs to $W^{1, \infty}(0, T ; X)$, which shows that the functions $u^{*}$ and $\varphi^{*}$ have the regularity expressed in (38), (39).

Uniqueness: The uniqueness of the solution is the result of the uniqueness of the fixed point of the operator $\Lambda$, given by the Lemma 5.4. Moreover, let $(u, \varphi, \beta)$ the solution to the Problem VP satisfy (38)-(40). Since by Lemma 5.2 this problem has a unique solution denoted $\left(u_{\beta}, \varphi_{\beta}\right)$, we obtain

$$
u=u_{\beta}, \quad \varphi=\varphi_{\beta} .
$$

Then, we replace $u=u_{\beta}$ in (36) and use the initial condition (37) to see that $\beta$ is a solution to Problem $\mathbf{V} \mathbf{P}_{\beta}^{\theta}$. Since by Lemma 5.3 this last problem has a unique solution denoted $\theta_{\beta}$, we find

$$
\beta=\theta_{\beta} .
$$

We use now (59) to see that $\Lambda \beta=\beta$, i.e., $\beta$ is a fixed point of the operator $\Lambda$. It follows now from Lemma 5.4 that

$$
\beta=\beta^{*}
$$

The uniqueness of the solution is a consequence of (63)-(65).

\section{Fully Discrete Approximations: Error Estimates}

We now introduce a finite element method to approximate solutions of Problem VP and derive an error estimate on them. The discretization of (34)-(37) is done as follows. First, We consider finite dimensional spaces $V^{h} \subset V, W^{h} \subset W$ and $B^{h} \subset L^{2}\left(\Gamma_{3}\right)$, approximating the spaces $V, W$ and $L^{2}\left(\Gamma_{3}\right)$, respectively. Here, $h>0$ denotes the spacial discretization parameter. Moreover, let $U^{h}$ be the discrete convex set of admissible displacement. Secondly, the time derivatives are discretized by using a uniform partition of $[0, T]$, denoted by $0=t_{0}<t_{1}<\cdots<t_{N}=T$. Let $k$ be the time step size, $k=T / N$, and for a continuous function $f(t)$ let $f_{n}=f\left(t_{n}\right)$. Finally, for a sequence $\left\{w_{n}\right\}_{n=0}^{N}$, we denote by $\delta w_{n}=\left(w_{n}-w_{n-1}\right) / k$ the finite differences. In this section, no summation is assumed over a repeated index and $c$ denotes a positive constant which depends 
on the problem data, but is independent of the discretization parameters, $h$ and $k$. Thus, using the backward Euler scheme, the fully discrete approximation of Problem VP is the following.

Problem $V P^{h k}$ : Find a discrete displacement field $u^{h k}=\left\{u_{n}^{h k}\right\}_{n=1}^{N} \subset V^{h}$, a discrete electric potential $\varphi^{h k}=\left\{\varphi_{n}^{h k}\right\}_{n=1}^{N} \subset W^{h}$ and a discrete bonding field $\beta^{h k}=\left\{\beta_{n}^{h k}\right\}_{n=1}^{N} \subset B^{h}$ such that $\beta_{0}^{h k}=\beta_{0}^{h}$ and for all $n=1, \ldots, N$

$$
\begin{aligned}
& \left\{\begin{array}{l}
\left(\mathcal{F} \varepsilon\left(u_{n}^{h k}\right), \varepsilon\left(v^{h}-u_{n}^{h k}\right)\right)_{\mathcal{H}}+\left(\mathcal{E}^{*} \nabla \varphi_{n}^{h k}, \varepsilon\left(v^{h}-u_{n}^{h k}\right)\right)_{\mathcal{H}} \\
+j\left(\beta_{n}^{h k}, u_{n}^{h k}, v^{h}-u_{n}^{h k}\right) \geq\left(f_{n}, v^{h}-u_{n}^{h k}\right)_{V}, \\
\forall v^{h} \in U^{h},
\end{array}\right. \\
& \left(\mathcal{B} \nabla \varphi_{n}^{h k}, \nabla \psi^{h}\right)_{H}-\left(\mathcal{E} \varepsilon\left(u_{n}^{h k}\right), \nabla \psi^{h}\right)_{H}=\left(q_{n}, \psi^{h}\right)_{W} \quad \forall \psi^{h} \in W^{h}, \\
& \delta \beta_{n}^{h k}=H_{\mathrm{ad}}\left(\beta_{n-1}^{h k}, \zeta_{\beta_{n-1}^{h k}}, R\left(\left|u_{(n-1) \tau}^{h k}\right|\right)\right)
\end{aligned}
$$

and $\beta_{0}^{h}$ is an appropriate approximation of the initial condition $\beta_{0}$. We notice that the fully discrete Problem VP ${ }^{h k}$ can be seen as a coupled system of variational equations. Using classical results of nonlinear variational equations (see [16]), we obtain that Problem VPP $\mathbf{P}^{h k}$ admits a unique solution in $U^{h} \subset V, W^{h} \subset W$ and $B^{h} \subset L^{2}\left(\Gamma_{3}\right)$. Our interest in this section lies in estimating the numerical errors $\left\|u_{n}-u_{n}^{h k}\right\|_{V},\left\|\varphi_{n}-\varphi_{n}^{h k}\right\|_{W}$ and $\left\|\beta_{n}-\beta_{n}^{h k}\right\|_{L^{2}\left(\Gamma_{3}\right)}$. We have the following main error estimates result.

Theorem 6.1 Let the assumptions of Theorem 4.1 hold. Let $(u, \varphi, \beta)$ and $\left(u_{n}^{h k}, \varphi_{n}^{h k}, \beta_{n}^{h k}\right)$ denote the solution to Problems VP and $V P^{h k}$, respectively. Then, the following error estimates hold true for all $v^{h}=\left\{v_{j}^{h}\right\}_{j=1}^{N} \subset U^{h}$ and $\psi^{h}=\left\{\psi_{j}^{h}\right\}_{j=1}^{N} \subset W^{h}$,

$$
\begin{aligned}
& \max _{1 \leq n \leq N}\left\{\left\|u_{n}-u_{n}^{h k}\right\|_{V}^{2}+\left\|\varphi_{n}-\varphi_{n}^{h k}\right\|_{W}^{2}+\left\|\beta_{n}-\beta_{n}^{h k}\right\|_{L^{2}\left(\Gamma_{3}\right)}^{2}\right\} \\
& \leq c\left(\begin{array}{l}
\max _{1 \leq n \leq N}\left\|\varphi_{n}-\psi_{n}^{h}\right\|_{W}^{2}+\max _{1 \leq n \leq N}\left\|u_{n}-v_{n}^{h}\right\|_{V}^{2} \\
+\left\|\beta_{0}-\beta_{0}^{h}\right\|_{L^{2}\left(\Gamma_{3}\right)}^{2}+\sum_{j=1}^{N} k\left[\left\|\beta_{j-1}-\beta_{j-1}^{h k}\right\|_{L^{2}\left(\Gamma_{3}\right)}^{2}\right] \\
+k\left(\|u\|_{H^{2}(0, T ; V)}+\|\varphi\|_{H^{2}(0, T ; W)}+\|\beta\|_{H^{2}\left(0, T ; L^{2}\left(\Gamma_{3}\right)\right)}\right) .
\end{array}\right)
\end{aligned}
$$

Proof First, let us obtain an error estimate on the electric potential. We proceed as in [3] where further details can be found. Taking (35) at time $t=t_{n}$ for $\psi=\psi^{h} \in W^{h}$ and subtracting it from (67), it follows that

$$
\left(\mathcal{B} \nabla\left(\varphi_{n}-\varphi_{n}^{h k}\right), \nabla \psi^{h}\right)_{H}-\left(\mathcal{E} \varepsilon\left(u_{n}-u_{n}^{h k}\right), \nabla \psi^{h}\right)_{H}=0 \quad \forall \psi^{h} \in W^{h} .
$$

Thus, we have

$$
\begin{aligned}
& \left(\mathcal{B} \nabla\left(\varphi_{n}-\varphi_{n}^{h k}\right), \nabla\left(\varphi_{n}-\varphi_{n}^{h k}\right)\right)_{H}-\left(\mathcal{E} \varepsilon\left(u_{n}-u_{n}^{h k}\right), \nabla\left(\varphi_{n}-\varphi_{n}^{h k}\right)\right)_{H} \\
& \quad=\left(\mathcal{B} \nabla\left(\varphi_{n}-\varphi_{n}^{h k}\right), \nabla\left(\varphi_{n}-\psi^{h}\right)\right)_{H}-\left(\mathcal{E} \varepsilon\left(u_{n}-u_{n}^{h k}\right), \nabla\left(\varphi_{n}-\psi^{h}\right)\right)_{H},
\end{aligned}
$$

for all $\psi^{h} \in W^{h}$. Applying the inequality

$$
a b \leq \epsilon a^{2}+\frac{1}{4 \epsilon} b^{2}, \quad a, b, \epsilon \in \mathbb{R}, \quad \epsilon>0
$$

we obtain

$$
\left\|\varphi_{n}-\varphi_{n}^{h k}\right\|_{W}^{2} \leq c\left(\left\|u_{n}-u_{n}^{h k}\right\|_{V}^{2}+\left\|\varphi_{n}-\psi^{h}\right\|_{W}^{2}\right) \quad \forall \psi^{h} \in W^{h} .
$$

Secondly, we proceed now to estimate the numerical errors on the displacement field. We rewrite variational equation (34) at time $t=t_{n}$ for $v=u_{n}^{h k} \in U^{h}$ to obtain

$$
\left\{\begin{array}{l}
\left(\mathcal{F}_{\varepsilon}\left(u_{n}\right), \varepsilon\left(u_{n}-u_{n}^{h k}\right)\right)_{\mathcal{H}}+\left(\mathcal{E}^{*} \nabla \varphi_{n}, \varepsilon\left(u_{n}-u_{n}^{h k}\right)\right)_{\mathcal{H}} \\
+j\left(\beta_{n}, u_{n}, u_{n}-u_{n}^{h k}\right) \leq\left(f_{n}, u_{n}-u_{n}^{h k}\right)_{V}, \quad \forall v^{h} \in U^{h}
\end{array}\right.
$$


Therefore, after some algebraic manipulations, we have

$$
\left\{\begin{array}{l}
\left(-\mathcal{F} \varepsilon\left(u_{n}^{h k}\right), \varepsilon\left(u_{n}-u_{n}^{h k}\right)\right)_{\mathcal{H}}+\left(-\mathcal{E}^{*} \nabla \varphi_{n}^{h k}, \varepsilon\left(u_{n}-u_{n}^{h k}\right)\right)_{\mathcal{H}} \\
-j\left(\beta_{n}^{h k}, u_{n}^{h k}, u_{n}-u_{n}^{h k}\right) \leq\left(\mathcal{F} \varepsilon\left(u_{n}^{h k}\right), \varepsilon\left(v^{h}-u_{n}\right)\right)_{\mathcal{H}}+\left(\mathcal{E}^{*} \nabla \varphi_{n}^{h k}, \varepsilon\left(v^{h}-u_{n}\right)\right)_{\mathcal{H}} \\
+j\left(\beta_{n}^{h k}, u_{n}^{h k}, u_{n}-u_{n}^{h k}\right) \leq\left(\mathcal{F} \varepsilon\left(u_{n}^{h k}\right), \varepsilon\left(v^{h}-u_{n}\right)\right)_{\mathcal{H}}+\left(\mathcal{E}^{*} \nabla \varphi_{n}^{h k}, \varepsilon\left(v^{h}-u_{n}\right)\right)_{\mathcal{H}} \\
+j\left(\beta_{n}^{h k}, u_{n}^{h k}, v^{h}-u_{n}\right)+\left(f_{n},-v^{h}+u_{n}^{h k}\right)_{V}, \quad \forall v^{h} \in U^{h}
\end{array}\right.
$$

We add inequalities (72) and (73) and we obtain

$$
\left\{\begin{array}{l}
\left(\mathcal{F} \varepsilon\left(u_{n}\right)-\mathcal{F}_{\varepsilon}\left(u_{n}^{h k}\right), \varepsilon\left(u_{n}-u_{n}^{h k}\right)\right)_{\mathcal{H}}+\left(\mathcal{E}^{*} \nabla\left(\varphi_{n}-\varphi_{n}^{h k}\right), \varepsilon\left(u_{n}-u_{n}^{h k}\right)\right)_{\mathcal{H}} \\
+j\left(\beta_{n}-\beta_{n}^{h k}, u_{n}-u_{n}^{h k}, u_{n}-u_{n}^{h k}\right) \leq\left(\mathcal{F} \varepsilon\left(u_{n}^{h k}\right), \varepsilon\left(v^{h}-u_{n}\right)\right)_{\mathcal{H}} \\
+\left(\mathcal{E}^{*} \nabla \varphi_{n}^{h k}, \varepsilon\left(v^{h}-u_{n}\right)\right)_{\mathcal{H}}+j\left(\beta_{n}^{h k}, u_{n}^{h k}, v^{h}-u_{n}\right) \\
-\left(f_{n}, v^{h}-u_{n}\right)_{V} \quad \forall v^{h} \in U^{h}
\end{array}\right.
$$

hence, we obtain

$$
\left\{\begin{array}{l}
\left(\mathcal{F} \varepsilon\left(u_{n}-u_{n}^{h k}\right), \varepsilon\left(u_{n}-u_{n}^{h k}\right)\right)_{\mathcal{H}}+\left(\mathcal{E}^{*} \nabla\left(\varphi_{n}-\varphi_{n}^{h k}\right), \varepsilon\left(u_{n}-u_{n}^{h k}\right)\right)_{\mathcal{H}} \\
+j\left(\beta_{n}-\beta_{n}^{h k}, u_{n}-u_{n}^{h k}, u_{n}-u_{n}^{h k}\right) \leq\left(\mathcal{F} \varepsilon\left(u_{n}^{h k}-u_{n}\right), \varepsilon\left(v^{h}-u_{n}\right)\right)_{\mathcal{H}} \\
+\left(\mathcal{E}^{*} \nabla\left(\varphi_{n}^{h k}-\varphi_{n}\right), \varepsilon\left(v^{h}-u_{n}\right)\right)_{\mathcal{H}}+j\left(\beta_{n}-\beta_{n}^{h k}, u_{n}^{h k}-u_{n}, v^{h}-u_{n}\right) \\
+Z\left(u_{n}, \varphi_{n}, \beta_{n}, v^{h}-u_{n}\right)
\end{array}\right.
$$

where we denote

$$
\begin{aligned}
& Z\left(u_{n}, \varphi_{n}, \beta_{n}, v^{h}-u_{n}\right)=\left(\mathcal{F} \varepsilon\left(u_{n}\right), \varepsilon\left(v^{h}-u_{n}\right)\right)_{\mathcal{H}}+\left(\mathcal{E}^{*} \nabla \varphi_{n}, \varepsilon\left(v^{h}-u_{n}\right)\right)_{\mathcal{H}} \\
& \quad+j\left(\beta_{n}, u_{n}, v^{h}-u_{n}\right)-\left(f_{n}, v^{h}-u_{n}\right)_{V} .
\end{aligned}
$$

From properties (45) and (46), we have

$$
j\left(\beta_{n}-\beta_{n}^{h k}, u_{n}-u_{n}^{h k}, u_{n}-u_{n}^{h k}\right) \leq c\left\|u_{n}-u_{n}^{h k}\right\|_{V}^{2}\left\|\beta_{n}-\beta_{n}^{h k}\right\|_{L^{2}\left(\Gamma_{3}\right)}
$$

and

$$
j\left(\beta_{n}-\beta_{n}^{h k}, u_{n}-u_{n}^{h k}, v^{h}-u_{n}^{h k}\right) \leq c\left\|u_{n}-u_{n}^{h k}\right\|_{V}\left\|v^{h}-u_{n}^{h k}\right\|_{V}\left\|\beta_{n}-\beta_{n}^{h k}\right\|_{L^{2}\left(\Gamma_{3}\right)} .
$$

Using repeatedly Inequality (75) and properties (23), and (70) after easy calculations it follows that

$$
\left\{\begin{array}{l}
\left\|u_{n}-u_{n}^{h k}\right\|_{V}^{2} \leq c\left[\left\|\varphi_{n}-\varphi_{n}^{h k}\right\|_{W}^{2}+\left\|\beta_{n}-\beta_{n}^{h k}\right\|_{L^{2}\left(\Gamma_{3}\right)}^{2}\right. \\
\left.+\left\|v^{h}-u_{n}\right\|_{V}^{2}+Z\left(u_{n}, \varphi_{n}, \beta_{n}, v^{h}-u_{n}\right)\right], \quad \forall v^{h} \in U^{h}
\end{array}\right.
$$

here and below, we assume that the estimation of error of the field of adhesion is small and is denoted as

$$
\delta \beta_{n}=\left(\beta_{n}-\beta_{n-1}\right) / k .
$$

Keeping in mind that

$$
\beta_{n}^{h k}=\sum_{j=1}^{n} k H_{\mathrm{ad}}\left(\beta_{j-1}^{h k}, \zeta_{\beta_{j-1}^{h k}}, R\left(\left|u_{(j-1) \tau}^{h k}\right|\right)\right),
$$

we subtract it from (68) to obtain

$$
\left\|\beta_{n}-\beta_{n}^{h k}\right\|_{L^{2}\left(\Gamma_{3}\right)} \leq c\left(\left\|\beta_{0}-\beta_{0}^{h}\right\|+\sum_{j=1}^{n} k\left(\left\|\beta_{j-1}-\beta_{j-1}^{h k}\right\|\right)+I_{n}\right),
$$

where

$$
I_{n}=\int_{0}^{t_{n}} H_{\mathrm{ad}}\left(\beta(s), \zeta_{\beta}(s), R\left(\left|u_{\tau}(s)\right|\right)\right) \mathrm{d} s-\sum_{j=1}^{n} k H_{\mathrm{ad}}\left(\beta_{j-1}, \zeta_{\beta_{j-1}}, R\left(\left|u_{(j-1) \tau}\right|\right)\right) .
$$


Then, combining inequalities (71) and (75) and taking into account the estimation (76), we then obtain

$$
\left\{\begin{array}{l}
\left\|u_{n}-u_{n}^{h k}\right\|_{V}^{2}+\left\|\varphi_{n}-\varphi_{n}^{h k}\right\|_{W}^{2}+\left\|\beta_{n}-\beta_{n}^{h k}\right\|_{L^{2}\left(\Gamma_{3}\right)}^{2} \leq \\
\left\|v_{n}^{h}-u_{n}\right\|_{V}^{2}+\left\|\varphi_{n}-\psi^{h}\right\|_{W}^{2}+\left\|\beta_{0}-\beta_{0}^{h}\right\|+\sum_{j=1}^{n} k\left(\left\|\beta_{j-1}-\beta_{j-1}^{h k}\right\|\right)+I_{n} \\
+Z\left(u_{n}, \varphi_{n}, \beta_{n}, v^{h}-u_{n}\right) .
\end{array}\right.
$$

From Theorem 4.1, we have $u \in H^{2}(0, T ; V)$ and, therefore, it is easy to check that

$$
I_{n} \leq k\left(\|u\|_{H^{2}(0, T ; V)}+\|\beta\|_{H^{2}\left(0, T ; L^{2}\left(\Gamma_{3}\right)\right)}\right) .
$$

Applying a discrete version of Gronwall's inequality (see [12]) for details, we then obtain Inequality (69).

We notice that the above error estimates are the basis for the analysis of the convergence rate of the algorithm. Thus, let $\Omega$ compatible with the partition of the boundary $\Gamma=\partial \Omega$ into $\Gamma_{1}, \Gamma_{2}$ and $\Gamma_{3}$ on the one hand, and into $\Gamma_{a}$ and $\Gamma_{b}$, on the other. Let $V^{h}$ and $W^{h}$ consist of continuous and piecewise affine functions; that is,

$$
\begin{aligned}
& V^{h}=\left\{v^{h} \in[C(\bar{\Omega})]^{d} ; v_{\mid T r}^{h} \in\left[P_{1}(\operatorname{Tr})\right]^{d} \quad \operatorname{Tr} \in \mathfrak{T}^{h}, v^{h}=0 \text { on } \Gamma_{1}\right\}, \\
& W^{h}=\left\{\psi^{h} \in[C(\bar{\Omega})] ; \psi_{\mid T r}^{h} \in P_{1}(\operatorname{Tr}) \operatorname{Tr} \in \mathfrak{T}^{h}, \psi^{h}=0 \text { on } \Gamma_{a}\right\}, \\
& B^{h}=\left\{\beta^{h} \in\left[C\left(\overline{\Gamma_{3}}\right)\right] ; \beta_{\mid T r}^{h} \in\left[P_{1}(\operatorname{Tr})\right] \operatorname{Tr} \in \mathfrak{T}^{h}, \beta^{h}=0 \text { on } \Gamma_{3}\right\},
\end{aligned}
$$

where $P_{1}(T r)$ represents the space of polynomials of global degree less or equal to one in $\operatorname{Tr}$. Assume that the discrete initial conditions $\beta_{0}^{h}$ is obtained by

$$
\beta_{0}^{h}=\Pi^{h} \beta_{0}
$$

where $\Pi^{h}=\left(\pi^{h}\right)_{i=1}^{d}:[C(\bar{\Omega})]^{d} \rightarrow V^{h}$ and $\pi^{h}$ is the standard finite element interpolation operator (see, e.g., [10]). Then, we have the following corollary which states the linear convergence of the algorithm under suitable regularity condition.

Corollary 6.2 Let the assumptions of Theorem 4.1 hold. Let $(u, \varphi, \beta)$ and $\left(u_{n}^{h k}, \varphi_{n}^{h k}, \beta_{n}^{h k}\right)$ denote the solutions to Problems VP and V $P^{h k}$, respectively, and let the discrete initial conditions be given by (81). Under the following regularity conditions:

$$
u \in C\left([0, T] ;\left[H^{2}(\Omega)\right]^{d}\right), \quad \varphi \in C\left([0, T] ; H^{2}(\Omega)\right), \quad \beta \in C^{1}\left([0, T] ;\left[H^{2}\left(\Gamma_{3}\right)\right]\right) .
$$

the linear convergence of the algorithm is achieved; that is, there exists a positive constant $c>0$, independent of the discretization parameters $h$ and $k$, such that

$$
\max _{1 \leq n \leq N}\left\{\left\|u_{n}-u_{n}^{h k}\right\|_{V}+\left\|\varphi_{n}-\varphi_{n}^{h k}\right\|_{W}+\left\|\beta_{n}-\beta_{n}^{h k}\right\|_{L^{2}\left(\Gamma_{3}\right)}\right\} \leq c(h+k) .
$$

Proof We have the following approximation properties of the finite element spaces $V^{h}, W^{h}$ and $B^{h}$ [10],

$$
\begin{aligned}
& \max _{1 \leq n \leq N} \inf _{\psi_{n}^{h} \in W^{h}}\left\|\varphi_{n}-\psi_{n}^{h}\right\|_{W} \leq \operatorname{ch}\|\varphi\|_{C\left([0, T] ; H^{2}(\Omega)\right)}, \\
& \max _{1 \leq n \leq N} \inf _{v_{n}^{h} \in V^{h}}\left\|u_{n}-v_{n}^{h}\right\|_{V} \leq \operatorname{ch}\|u\|_{C\left([0, T] ;\left[H^{2}(\Omega)\right]^{d}\right)} .
\end{aligned}
$$

Moreover, from the definition of the finite element interpolation operator $\Pi^{h}$, it follows that

$$
\left\|\beta_{0}-\beta_{0}^{h}\right\|_{L^{2}\left(\Gamma_{3}\right)} \leq \operatorname{ch}\|\beta\|_{C^{1}\left([0, T] ;\left[H^{2}\left(\Gamma_{3}\right)\right]\right)},
$$

and combining the previous estimates, (69) and (76) it leads to Inequality (82).

Open Access This article is distributed under the terms of the Creative Commons Attribution Noncommercial License which permits any noncommercial use, distribution, and reproduction in any medium, provided the original author(s) and source are credited. 


\section{References}

1. Andrews, K.T.; Chapman, L.; Fernàndez, J.R.; Shillor, M.; Vanerian, L.; Van Houten, T.: A membrane in adhesive contact. SIAM J. Appl. Math. 64, 152-169 (2003)

2. Andrews, K.T.; Shillor, M.: Dynamic adhesive contact of a membrane. Adv. Math. Sci. Appl. 13(1), 343-356 (2003)

3. Barboteu, M.; Ramon, J.; Fernàndez, J.R.; Ouafik, Y.: Numerical analysis of a frictionless viscoelastic-piezoelectric contact problem. M2AN Math. Model. Numer. Anal. 42(4), 667-682 (2008)

4. Batra, R.C.; Yang, J.S.: Saint-Venant's principle in linear piezoelectricity. J. Elast. 38(2), 209-218 (1995)

5. Barboteu, M.; Fernandez, J.R.; Ouafik, Y.: Numerical analysis of two frictionless elastic-piezoelectric contact problems. J. Math. Anal. Appl. 339(2), 905-917 (2008)

6. Bisegna, P.; Lebon, F.; Maceri, F.: The unilateral frictional contact of a piezoelectric body with a rigid support. Contact Mechanics (Praia da Consolacão, 2001) In: Martins, J.A.C.; Montereiro Marques, M.D.P. (eds.): Solid Mech. Appl, vol. 103. Kluwer Academic, Dordrecht, pp. 47-354 (2002)

7. Buchukuri, T.; Gegelia, T.: Some dynamic problems of the theory of electroelasticity. Mem. Differ. Equ. Math. Phys. 10, 1-53 (1997)

8. Chau, O.; Fernàndez, J.R.; Shillor, M.; Sofonea, M.: Variational and numerical analysis of a quasistatic viscoelastic contact problems with adhesion. J. Comput. Appl. Math. 159, 431-465 (2003)

9. Chau, O.; Shillor, M.; Sofonea, M.: Dynamic frictionless contact with adhesion. J. Appl. Math. Phys. (ZAMP) 55, 32-47 (2004)

10. Ciarlet, P.G.; Lions, J.L. (eds.): The finite element method for elliptic problems. In: Ciarlet, P.G., Lions, J.L. (eds.) Handbook of Numerical Analysis, vol. II. North Holland, pp. 17-352 (1991)

11. Drabla, S.; Zellagui, Z.: Analyse of A Eectro-Elastic Contact Problem With Friction And Adhesion Studia Universitatis. "Babes-Bolyai", Mathematica, LIV(1) (2009)

12. Han, W.; Sofonea, M.: Quasistatic Contact Problems in Viscoelasticity and Viscoplasticity. American Mathematical Societyinternational Press, Providence (2002)

13. Ikeda, T.: Fundamentals of Piezoelectricity. Oxford University Press, Oxford (1990)

14. Jianu, L.; Sillor, M.; Sofonea, M.: A viscoelastic frictionless contact problem with adhesion. Appl. Anal. 80, 233-255 (2001)

15. Kikuchi, N.; Oden, J.T.: Contact problem in elasticity: A study of variational inequation and finite element methods. SIAM, Philadelphia (1988)

16. Klarbring, A.; Mikelic, A.; Shillor, M.: Frictional contact problems with normal compliance. Int. J. Eng. Sci. 26, 811-832 (1988)

17. Sofonea, M.; Matei, A.: A quasistatic frictionless contact problem with normal compliance. Ann. Univ. Craiova. Math-info XXVII, 43-56 (2000) 